Check for updates

Cite this: RSC Adv., 2019, 9, 2870

Received 5th November 2018

Accepted 17th January 2019

DOI: 10.1039/c8ra09143a

rsc.li/rsc-advances

\section{Structure evolution of chromium-doped boron clusters: toward the formation of endohedral boron cages $\uparrow$}

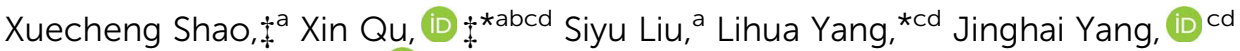 \\ Xiaohui Liu, ${ }^{e}$ Xin Zhong, (D) *cd ${ }^{*}$ Shuai Sun, ${ }^{f}$ G. Vaitheeswaran ${ }^{9}$ and Jian Lv*ab
}

\begin{abstract}
The electron-deficient nature of boron endows isolated boron clusters with a variety of interesting structural and bonding properties that can be further enriched through metal doping. In the current work, we report the structural and electronic properties of a series of chromium-doped boron clusters. The global minimum structures for $\mathrm{CrB}_{n}$ clusters with an even number of $n$ ranging from 8 to 22 are proposed through extensive first-principles swarm-intelligence structure searches. Half-sandwich structures are found to be preferred for $\mathrm{CrB}_{8}, \mathrm{CrB}_{10}, \mathrm{CrB}_{12}$ and $\mathrm{CrB}_{14}$ clusters and to transform to a drum-like structure at $\mathrm{CrB}_{16}$ cluster. Endohedral cage structures with the $\mathrm{Cr}$ atom located at the center are energetically most favorable for $\mathrm{CrB}_{20}$ and $\mathrm{CrB}_{22}$ clusters. Notably, the endohedral $\mathrm{CrB}_{20}$ cage has a high symmetry of $D_{2 d}$ and a large HOMO-LUMO gap of $4.38 \mathrm{eV}$, whose stability is attributed to geometric fit and formation of an 18-electron closed-shell configuration. The current results advance our understanding of the structure and bonding of metal-doped boron clusters.
\end{abstract}

\section{Introduction}

Boron is an element of fascinating structural and chemical complexity, leading to topics of considerable interest in chemistry. It has three valence electrons that are deficient compared with the four valence orbitals, and it prefers to share rather than donate the valence electrons. These characteristics make it difficult for B to achieve filled octets through classical 2c-2e bonds, giving rise to a rich variety of structures along with electron-deficient multicentered bonds, both in its elemental form and chemical compounds. ${ }^{\mathbf{1}}$ Sixteen polymorphs have been discovered for bulk $\mathrm{B}$ with $\mathrm{B}_{12}$ icosahedron being a prevalent

${ }^{a}$ State Key Laboratory for Superhard Materials, College of Physics, Jilin University, Changchun, 130012, China.E-mail: quxin@calypso.cn

${ }^{b}$ College of Materials Science and Engineering, Jilin University, Changchun 130012, China

'Key Laboratory of Functional Materials Physics and Chemistry of the Ministry of Education, Jilin Normal University, Changchun 130103, China. E-mail: ylh@ calypso.cn

${ }^{d}$ National Demonstration Center for Experimental Physics Education, Jilin Normal University, Siping 136000, China

${ }^{e}$ Network Information Center, Supercomputing Center, University of Science and Technology of China, Hefei 230026, China

${ }^{f}$ Engineering Training Center, Institute of Mechanical Science and Engineering, Jilin University, Changchun, 130012, China

${ }^{8}$ Advanced Center of Research in High Energy Materials (ACRHEM), University of Hyderabad, Hyderabad, 500046, India

$\dagger$ Electronic supplementary information (ESI) available. See DOI: 10.1039/c8ra09143a

\$ Equally share the first authorship. motif. $^{2}$ 1D nanotubes ${ }^{3-5}$ and $2 \mathrm{D}$ sheets ${ }^{6-8}$ have been fabricated in which triangular planar $\mathrm{B}$ lattices with hexagonal holes are found to be energetically favorable. ${ }^{9}$

For 0D B clusters, the situation is even more interesting. Joint photoelectron spectroscopy and theoretical studies carried out over the past decades show that anionic $\mathrm{B}_{n}{ }^{-}$clusters up to $n$ $=38$ are planar or quasi-planar, ${ }^{\mathbf{1 0 , 1 1}}$ in which delocalized multicentered bonds are responsible for the stabilities. ${ }^{12}$ Theoretical calculations suggested a planar-to-tubular structure transition taking place at $\mathrm{B}_{20}$ for neutrals. ${ }^{13}$ Subsequently, combined collision cross section measurements and theoretical calculations confirmed the existence of tubular structures for cationic $\mathrm{B}_{n}{ }^{+}$clusters with $n=16-25 .{ }^{14}$ Most strikingly, the longsought B fullerene analogue (borospherenes) was first observed at $\mathrm{B}_{40}$ (ref. 15) after extensive theoretical investigations, ${ }^{\mathbf{1 6}-19}$ and a series of axially chiral borospherenes were subsequently identified at $\mathrm{B}_{39}{ }^{-}{ }^{20} \mathrm{~B}_{41}{ }^{+}$and $\mathrm{B}_{42}{ }^{2+} \cdot{ }^{21}$ For larger B clusters, recent theoretical studies have suggested quasi-planar, ${ }^{22,23}$ tubular, ${ }^{24}$ cage-like $^{25}$ and bilayered ${ }^{23}$ structures as ground states at certain sizes, and core-shell structures are generally expected to be energetically most favorable for $n>\sim 68 .{ }^{26-28}$

The structural diversity of B clusters can be further enriched through metal doping. Metal-centered monocyclic B rings can be formed by transition metal doping of small $\mathrm{B}_{8-10}$ anionic clusters ${ }^{29}$ in which $\mathrm{NbB}_{10}{ }^{-}$(ref. 30) and $\mathrm{TaB}_{10}{ }^{-}$(ref. 30) hold the highest coordination number of 10 in planar molecular species. Interesting half-sandwich structures have been found in $\mathrm{Co} / \mathrm{Rh}$ doped $\mathrm{B}_{12}$ anionic clusters, ${ }^{31}$ and drum-like structures have been observed in $\mathrm{CoB}_{16}{ }^{-},{ }^{32} \mathrm{MnB}_{16}{ }^{-}$(ref. 33) and $\mathrm{TaB}_{20}{ }^{-}$(ref. 34) 
clusters, in which the $\mathrm{TaB}_{20}{ }^{-}$cluster possesses the highest coordination number (20) heretofore known in chemistry. Motivated by the discovery of borospherenes in our previous work, we have designed symmetric endohedral B cages, such as $\mathrm{MnB}_{20}{ }^{+}{ }^{35} \mathrm{MoB}_{24}$ (ref. 36) and $\mathrm{WB}_{24}{ }^{36}$ whose stabilities are attributed to the formation of 18-electron closed-shell configurations, as well as geometric fits between the sizes of the transition metal atoms and the cavities of the B cages. It was further suggested that, by reasonable choice of the transition metal atom and number of B atoms, other-sized boron cages are also likely to be stabilized. Recently, a theoretical study found that even the small $\mathrm{B}_{12}$ cluster can show interesting transitions from quasi-planar to tubular and cage-like structures through interactions with lithium atoms, in which charge transfer plays a critical role. ${ }^{37}$

Metal doping has been proven to be an effective avenue to achieve intriguing structure motifs in B clusters. Given the large number of possible combinations between metal atoms and the number of B atoms yet to be investigated, it not unreasonable to expect more fascinating phenomena in this group of chemical species. As such, in this paper, we report systematic investigations on the structure and bonding of a series of chromium $(\mathrm{Cr})$ doped B clusters by means of the swarm-intelligent CALYPSO structure searching method and first-principles density functional calculations. Ground-state structures are proposed for $\mathrm{CrB}_{n}$ clusters with an even number of $n$ ranging from 8 to 22, revealing an intriguing transition from half-sandwich to drumlike and then endohedral cage-like structures. In particular, a symmetric $D_{2 \mathrm{~h}}$ endohedral cage is revealed as the ground-state structure for the $\mathrm{CrB}_{20}$ cluster. The rest of the manuscript is organized as follow. The second section describes the computational details. Section 3 presents the results and discussion, and the conclusions from the present results are given in Section 4 .

\section{Calculation details}

The unbiased structure searches of $\mathrm{CrB}_{n}$ clusters with an even number of $n$ ranging from 8 to 22 are based on the global minimization of the potential energy surfaces, merging $a b$ initio total energy calculations via the CALYPSO (Crystal structure AnaLYsis by Particle Swarm Optimization) package. ${ }^{38-41}$ Several major techniques are included in the algorithm to achieve high efficiency, e.g., point group symmetry constraints in structural generation, bond characterization matrix technique for fingerprinting structures, and a local version of the particle swarm optimization algorithm enabling simultaneous search in different energy funnels. ${ }^{39}$ Its validity has been manifested by successful identification of the ground-state structures for a large number of systems. More than 2000 trial structures were generated for each cluster.

During the structure searches, the underlying energy calculations and structure relaxations are carried out in the framework of density functional theory (DFT) with the PBE

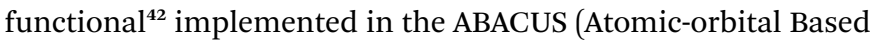
Ab initio Computation at USTC) package. ${ }^{43}$ ABACUS was developed to perform large-scale DFT simulations using linear combinations of atomic orbitals. ${ }^{\mathbf{4 4 , 4 5}}$ The recently developed systematically improvable optimized numerical atomic orbitals $^{\mathbf{4 4 , 4 5}}$ were found to be an excellent choice to describe various materials, such as molecules, crystalline solids, surfaces, and defects. ${ }^{43}$ The atomic orbitals basis set of $\mathrm{B}$ includes two $\mathrm{s}$, two $\mathrm{p}$ and one polarized d orbitals (2s2p1d), whereas the basis set of $\mathrm{Cr}$ includes four s, two p, two d and one polarized $\mathrm{f}$ orbitals (4s2p2d1f). The radii of the numerical atomic orbitals are set to 7 bohr for B and 8 bohr for $\mathrm{Cr}$ in the first-round local structure optimization, while 8 bohr for B and 9 bohr for $\mathrm{Cr}$ are used in the second-round local structure optimization. We adopt the SG15 Optimized Norm-Conserving Vanderbilt pseudopotentials, ${ }^{\mathbf{4 6}}$ and the energy cutoff for charge density is 240 Ry.

The low-lying isomers obtained from the structure searches were reoptimized at different spin states at $\mathrm{PBE0} / \mathrm{Cr} / \mathrm{Stuttgart} / \mathrm{B} /$ $6-311+\mathrm{G}^{*}$ level of theory using the Gaussian 09 Package. ${ }^{47}$ The calculation of harmonic vibrational frequencies ensures that the cluster geometries are true local minima on the potential energy surface (no imaginary frequencies obtained). At this step, singlet, triplet, quintet and septet states were considered for all of these even-number-electrons clusters. In a previous benchmark calculation, the PBE0 functional was confirmed to be suitable for describing the energy difference of isomers of medium-sized boron clusters (e.g., $\mathrm{B}_{20}$ ) compared to the highlevel $\operatorname{CCSD}(\mathrm{T})$ results. $^{28}$ The natural bond orbital (NBO) and adaptive natural density partitioning (AdNDP) analytical methods were carried out with the Multiwfn package ${ }^{48}$ in order to achieve a better understanding of the bonding mechanism.

\section{Results and discussion}

The global minimum structures for $\mathrm{CrB}_{n}(n=8,10,12,14,16$, 18, 20 and 22) clusters obtained from the current structure searches are depicted in Fig. 1. To facilitate understanding of the structures via visualization, top and side views are given along with their point group symmetries, spin multiplicity values $(M)$ and HOMO-LUMO energy gaps $\left(E_{\mathrm{g}}\right)$. Other low-lying isomers are shown in the ESI, Fig. S1-S8. $\dagger$ Generally, the effect of $\mathrm{Cr}$ doping on the structures of B clusters gradually is enhanced as the number of B atoms increases. For small-sized $\mathrm{CrB}_{n}(n=8,10$ and 12) clusters, the B structures are similar to those in bare B clusters, while larger $\mathrm{CrB}_{n}$ clusters exhibit B structures different from the corresponding bare B clusters. This leads to interesting transitions from half-sandwich to drum-like and then to endohedral cage-like structures as the number of B atoms increases. High spin states (triplet and quintet states) are preferred for small-sized $\mathrm{CrB}_{n}$ clusters with $n$ $<14$, and the magnetism is completely quenched for $\mathrm{CrB}_{n}$ clusters with $n \geq 16$.

\section{1 $\mathrm{CrB}_{8}, \mathrm{CrB}_{10}, \mathrm{CrB}_{12}$ and $\mathrm{CrB}_{14}$ clusters with half-sandwich structures}

As depicted in Fig. 1, small-sized $\mathrm{CrB}_{n}$ clusters with $n=8,10,12$ and 14 exhibit half-sandwich structures, whereas quasi-planar or bowl-like $\mathrm{B}_{n}$ moieties are coordinated to the $\mathrm{Cr}$ atom. High 

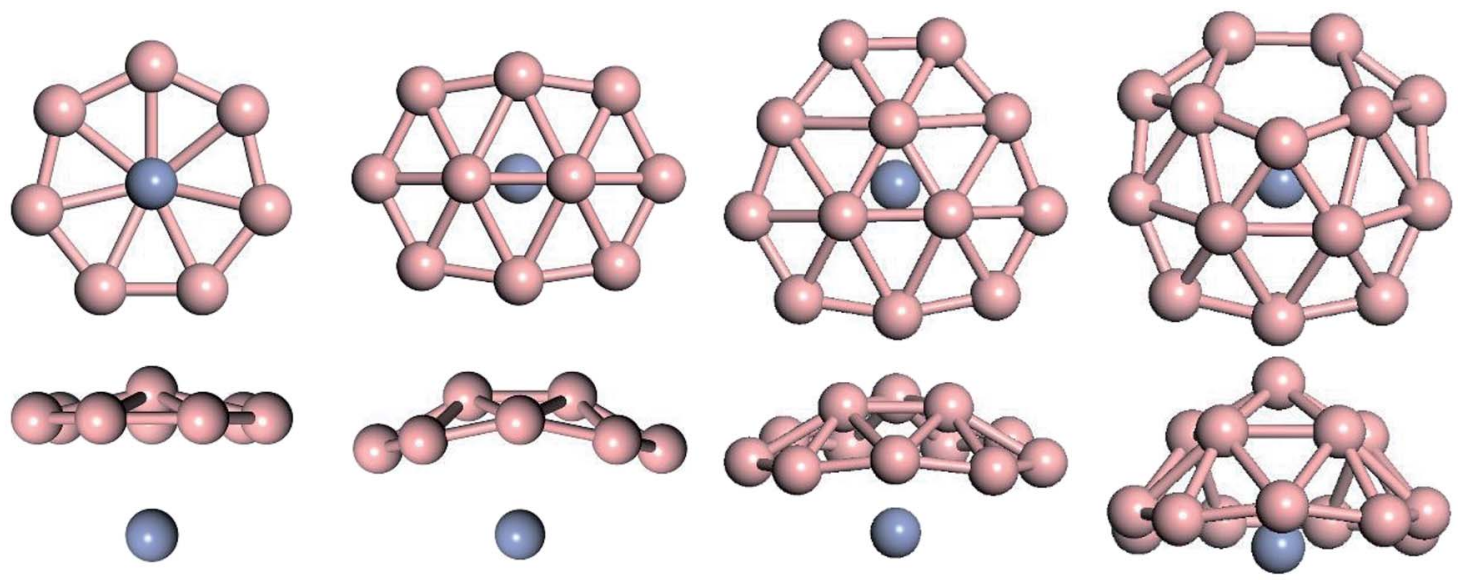

\section{$\mathrm{CrB}_{8} C_{7 v} \mathrm{M}=5$ \\ $\mathrm{Eg}=2.00 \mathrm{eV}$}
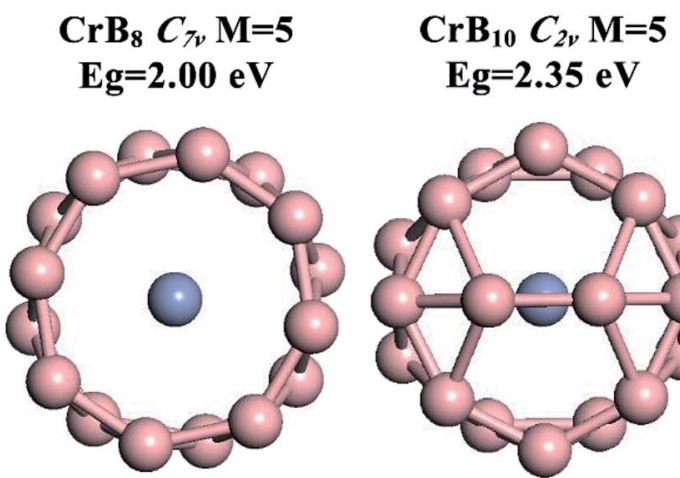

$\mathrm{CrB}_{12} C_{3 v} \mathrm{M}=5$

$\mathrm{Eg}=2.04 \mathrm{eV}$
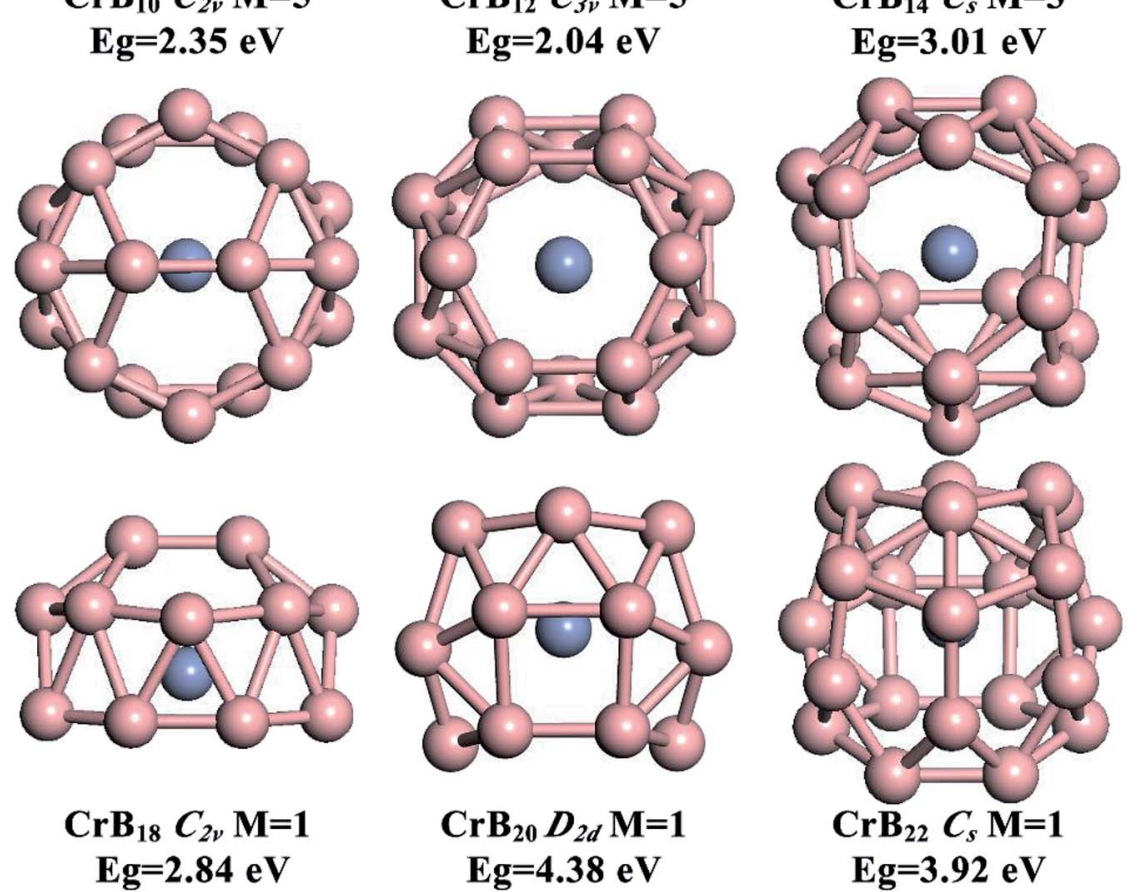

\section{$\mathrm{CrB}_{16} C_{2 v} \mathrm{M}=1$ \\ $\mathrm{Eg}=1.49 \mathrm{eV}$}
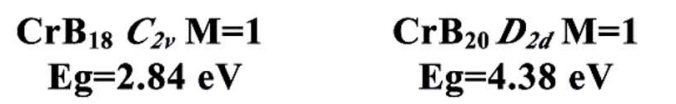

$\mathrm{Eg}=3.92 \mathrm{eV}$

Fig. 1 The lowest-energy ground-state isomers of $\mathrm{CrB}_{n}(n=8,10,12,14,16,18,20$ and 22) clusters derived from the global minimum structure search. For each system, two sides of the views are given. For each structure, the point group symmetry, spin multiplicity (M) and LUMO-HOMO gap $\left(E_{\mathrm{g}}\right)$ are indicated.

spin states are found to be the ground states for these globalminimum structures (quintets for $\mathrm{CrB}_{8}, \mathrm{CrB}_{10}, \mathrm{CrB}_{12}$, and triplets for $\mathrm{CrB}_{14}$ ). Other low-lying isomers are given in Fig. S1-S8 in the ESI. $\dagger$ Spin density distributions shown in ESI, Fig. S9 $\dagger$ indicates that the magnetism mainly originates from the unpaired $3 \mathrm{~d}$ electrons of the $\mathrm{Cr}$ atom.

The B structures in $\mathrm{CrB}_{8}, \mathrm{CrB}_{10}$ and $\mathrm{CrB}_{12}$ are very similar to those in bare B clusters, which are quasi-planar with one, two and three interior B atoms surrounded by seven-, eight- and nine-membered $B$ rings, respectively. Due to the existence of the $\mathrm{Cr}$ atom, the interior B atoms in the B moieties display slight out-of-plane distortions. Note that, although $\mathrm{CrB}_{10}$ is isovalent to $\mathrm{NbB}_{10}{ }^{-}$and $\mathrm{TaB}_{10}{ }^{-}$, it does not adopt the metal-centered monocyclic $\mathrm{B}$ rings in $\mathrm{NbB}_{10}{ }^{-}$and $\mathrm{TaB}_{10}{ }^{-}$as the ground state. This may be due to the smaller size of the $\mathrm{Cr}$ atom $(1.39 \AA)$ compared with those of $\mathrm{Nb}(1.64 \AA)$ and $\mathrm{Ta}(1.70 \AA)$, which is not optimal for fitting the cavity of the ten-membered B ring. The half-sandwich structure of $\mathrm{CrB}_{12}$ is the same as those in experimental $\mathrm{CoB}_{12}{ }^{-}$and $\mathrm{RhB}_{12}{ }^{-}$, further indicating that the double aromatic $\mathrm{B}_{12}$ moiety is a promising inorganic ligand.

Inserting two $\mathrm{B}$ atoms into the $\mathrm{B}_{12}$ moiety in the $\mathrm{CrB}_{12}$ cluster leads to the formation of $\mathrm{CrB}_{14}$. The $\mathrm{B}_{14}$ moiety in $\mathrm{CrB}_{14}$ has a bowl-like structure with five interior $\mathrm{B}$ atoms surrounded by a nine-membered $\mathrm{B}$ ring, which is different from the structures of neutral or charged bare $\mathrm{B}_{14}$ clusters. ${ }^{10,49}$ In contrast to $\mathrm{CrB}_{8}$, $\mathrm{CrB}_{10}$ and $\mathrm{CrB}_{12}$, the $\mathrm{Cr}$ atom is half encircled by the bowl-like $\mathrm{B}_{14}$ moiety in $\mathrm{CrB}_{14}$, leading to enhanced $\mathrm{Cr}-\mathrm{B}$ interactions and partially quenched magnetism. The significant curving of the $\mathrm{B}$ moiety in $\mathrm{CrB}_{14}$ indicates the gradually enhanced effect of Cr doping on the structure evolution and onset of structure transition in $\mathrm{CrB}_{n}$ clusters. 
(a)

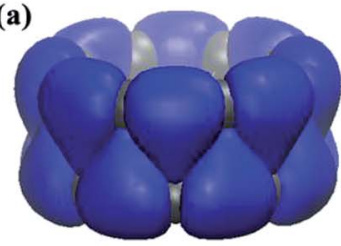

Sixteen 3c-2e B-B $\sigma$ bonds
ON=1.94 $|\mathrm{e}|$

(e)

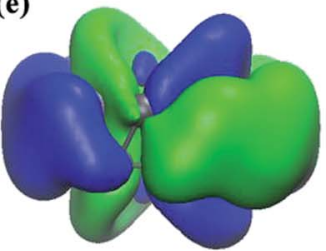

(b)

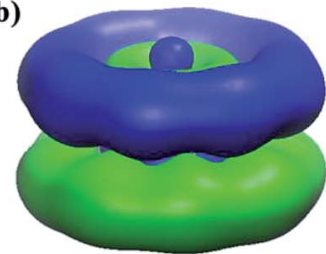

One 16c-2e $\sigma-\sigma$ bond $\mathrm{ON}=1.78|\mathrm{e}|$

(f)

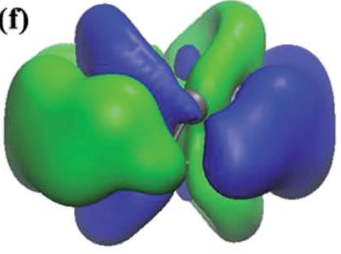

Two 16c-2e $\sigma+\sigma$ bonds

$\mathrm{ON}=1.74|\mathrm{e}|$

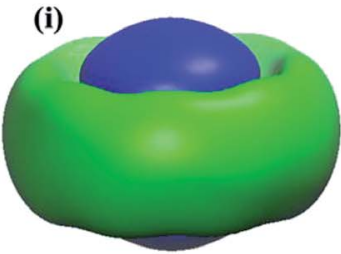

(j)

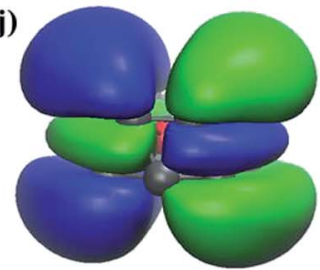

Three 16c-2e $\pi-\pi$ bonds $\mathrm{ON}=1.80-2.00|\mathrm{e}|$ (c)

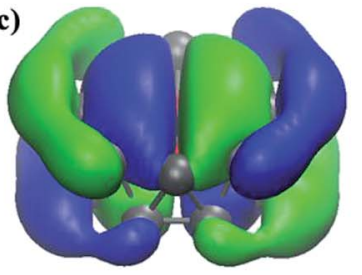

(d)

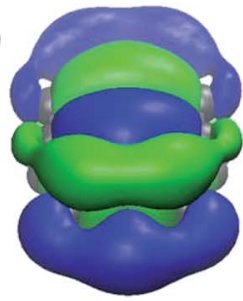

Two 17c-2e $\sigma-\sigma$ bonds $\mathrm{ON}=2.00|\mathrm{e}|$ (g)

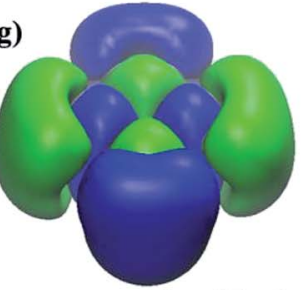

(h)

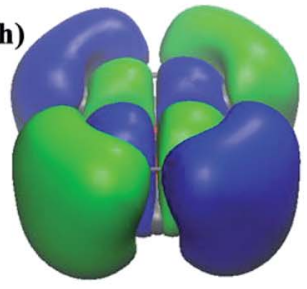

Two 17c-2e $\sigma+\sigma$ bonds $\mathrm{ON}=\mathbf{2 . 0 0}|\mathrm{e}|$

(k)

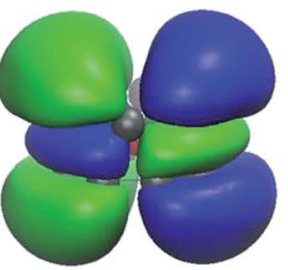

(l)

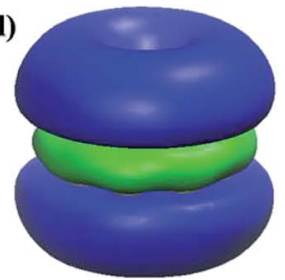

One 17c-2e $\pi-\pi$ bond $\mathrm{ON}=1.71|\mathrm{e}|$

Fig. 2 Images of the chemical bonding of $\mathrm{CrB}_{16}$ obtained from AdNDP analyses. ON stands for occupation number.

\subsection{The $\mathrm{CrB}_{16}$ cluster with a drum-like structure}

The well-known drum-like structure with the $\mathrm{Cr}$ atom located at the center of a $\mathrm{B}_{16}$ double-ring tube occurs with the $\mathrm{CrB}_{16}$ cluster, having a point group of $C_{2 \mathrm{v}}$ (Fig. 1). Within this structure, the magnetism is completely quenched due to the strong coordination interactions between the $\mathrm{Cr}$ atom and the $\mathrm{B}_{16}$ tube. This type of structure was initially observed in $\mathrm{CoB}_{16}{ }^{-}$(ref. 32) and $\mathrm{MnB}_{16}{ }^{-}$(ref. 33) by joint photoelectron spectroscopy measurement and $a b$ initio calculations. Later, studies found that larger $\mathrm{B}_{18}$ and $\mathrm{B}_{20}$ tubes can be stabilized by doping larger transition metal atoms ( $\mathrm{Rh}$ and $\mathrm{Ta}$ ), leading to drum-like $\mathrm{RhB}_{18}{ }^{-}$(ref. 50) and $\mathrm{TaB}_{20}{ }^{-34}$ respectively. Interestingly, recent theoretical studies have found that the smaller $\mathrm{B}_{14}$ tube can be formed by the doping of an Fe atom, ${ }^{51}$ though the size of Fe $(1.32 \AA)$ is larger than that of Co $(1.26 \AA)$. Thus, it seems that whether drum-like structures can be formed in metal-doped B clusters is closely related to the size of the doping atom and the cavity of the B tube, as well as the charge and spin states of the metal-doped B clusters.

The chemical bonding of the current drum-like $\mathrm{CrB}_{16}$ cluster was analyzed using the Adaptive Natural Density Partitioning $(\text { AdNDP })^{52}$ method, which is an extension of the Natural Bond Orbital method. ${ }^{53}$ AdNDP analyses can display both localized and delocalized bonding in molecules simultaneously, providing relatively simple bonding pictures for complicated molecular structures. ${ }^{33}$ The AdNDP analyses revealed a similar bonding character for $\mathrm{CrB}_{16}$ as those of $\mathrm{CoB}_{16}{ }^{-}$and $\mathrm{MnB}_{16}{ }^{-}$, where the 54 valence electrons in $\mathrm{CrB}_{16}$ can be divided into four bonding types as shown in Fig. 2. The occupation numbers (ON) of all the identified bonds range from 1.71 to $2.00|e|$. The first type represents localized bonds (Fig. 2a), which can be described in two manners: (1) as sixteen 3c-2e $\sigma$ bonds on the sixteen $\mathrm{B}_{3}$ triangles on the drum surface with $\mathrm{ON}=1.94|e|$ or (2) as sixteen $2 \mathrm{c}-2 \mathrm{e} \sigma$ bonds within the two $\mathrm{B}_{8}$ rings with $\mathrm{ON}=$ $1.73|e|$. In fact, the sixteen $3 \mathrm{c}-2 \mathrm{e} \sigma$ bonds can also be represented by sixteen $2 \mathrm{c}-2 \mathrm{e} \sigma$ bonds on the two $\mathrm{B}_{8}$ rings on the drum surface are shown in Fig. S10 in ESI. $\dagger$

The remaining three bonding types describe totally delocalized bonds (Fig. 2b-d, e-i and j-m) and account for bonding between the two $\mathrm{B}_{8}$ rings and between the $\mathrm{Cr}$ atom and the $\mathrm{B}_{16}$ tube. Following the previous work on $\mathrm{CoB}_{16}{ }^{-}$and $\mathrm{MnB}_{16}{ }^{-}$, the "+" sign is used to denote that the delocalized bonds between the two $\mathrm{B}_{8}$ rings overlap positively, while the “-” sign means a negative overlap. The second bonding type (Fig. 2b-d) consists of one $16 \mathrm{c}-2 \mathrm{e} \sigma-\sigma$ bond with $\mathrm{ON}=1.78|e|$ and two $17 \mathrm{c}-2 \mathrm{e} \sigma-$ $\sigma$ bonds with $\mathrm{ON}=2.00|e|$. The one $16 \mathrm{c}-2 \mathrm{e} \sigma-\sigma$ bond represents a bonding interaction within each $\mathrm{B}_{8}$ ring and an antibonding interaction between the two $\mathrm{B}_{8}$ rings, while the two $17 \mathrm{c}-2 \mathrm{e} \sigma-\sigma$ bonds represent mainly covalent bonding between Mn $\left(3 \mathrm{~d}_{x z}\right.$ and $\left.3 \mathrm{~d}_{y z}\right)$ and the $\mathrm{B}_{16}$ tube. The third bonding type (Fig. $2 \mathrm{e}-\mathrm{h}$ ) contains two $16 \mathrm{c}-2 \mathrm{e} \sigma+\sigma$ bonds with $\mathrm{ON}=1.74|e|$ and two $17 \mathrm{c}-2 \mathrm{e} \sigma+\sigma$ bonds with ONs $=2.00|e|$. The two $16 \mathrm{c}-2 \mathrm{e}$ bonds represent delocalized $\sigma$ bonding in the $\mathrm{B}_{16}$ frame, and the two $17 \mathrm{c}-2 \mathrm{e} \sigma+\sigma$ bonds represent covalent bonding between 


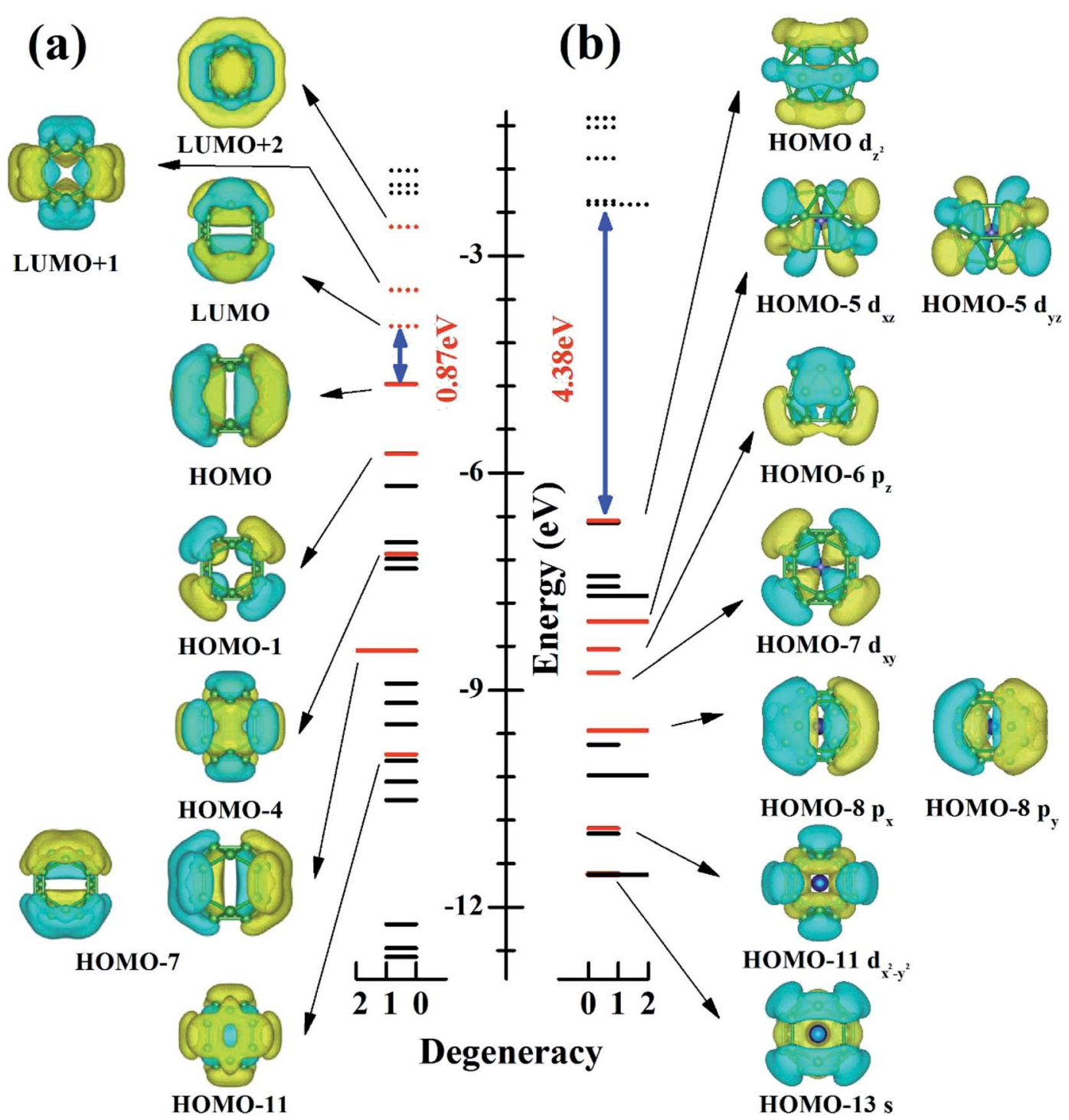

Fig. 3 Eigenvalue spectrum vs. electronic state degeneracy of (a) bare $D_{2 d}-B_{20}$ cage and (b) $D_{2 d}-C r B_{20}$. For each case, the HOMO-LUMO gap is indicated (in blue). The $\pi$-orbitals (red lines) (a) and the orbitals involving the 18 -electron closed-shell configuration (b) are shown.

$\operatorname{Cr}\left(3 \mathrm{~d}_{x y}\right.$ and $\left.3 \mathrm{~d}_{x^{2}-y^{2}}\right)$ and the $\mathrm{B}_{16}$ tube. The fourth bonding type consists of three $16 \mathrm{c}-2 \mathrm{e} \pi-\pi$ bonds and one $17 \mathrm{c}-2 \mathrm{e} \pi-\pi$ bond. These four bonds account for $\pi$ bonding interactions between the two $\mathrm{B}_{8}$ rings.

\subsection{Transition from drum-like to endohedral cage-like structures in $\mathrm{CrB}_{18}, \mathrm{CrB}_{20}$ and $\mathrm{CrB}_{22}$ clusters}

As depicted in Fig. 1, capping two B atoms on one side of the drum-like $\mathrm{CrB}_{16}$ structure leads to the global minimum structure of the $\mathrm{CrB}_{18}$ cluster, which can be seen as an intermediate structure between drum-like and endohedral cage-like structures. Notably, a symmetric endohedral cage with the $\mathrm{Cr}$ atom located at the center emerges as the global minimum structure for the $\mathrm{CrB}_{20}$ cluster. The structure is composed of twenty B triangles, four $\mathrm{B}$ quadrangles and two $\mathrm{B}$ hexagons in $D_{2 \mathrm{~d}}$ symmetry. It has the largest HOMO-LUMO gap of $4.38 \mathrm{eV}$ among $\mathrm{CrB}_{n}$ clusters considered in the current work. The high symmetry and large HOMO-LUMO gap imply highly degenerate electronic states and the potentially high chemical stability of the $\mathrm{CrB}_{20}$ cluster. Further addition of two B atoms into the $\mathrm{CrB}_{20}$ cluster also leads to an endohedral cage-like structure in the $\mathrm{CrB}_{22}$ cluster, suggesting that endohedral cage-like motifs may be prevalent for larger $\mathrm{CrB}_{n}$ clusters with $n>20$. However, the endohedral $\mathrm{CrB}_{22}$ cage is rather irregular, and its HOMO-LUMO gap $(3.92 \mathrm{eV})$ is smaller than that of $\mathrm{CrB}_{20}$.

Early theoretical studies have demonstrated metal doping as a viable route for stabilizing cage-like B structures. A series of transition-metal-centered endohedral $\mathrm{B}$ cages have been proposed, such as $\mathrm{FeB}_{18}, \mathrm{FeB}_{20}, \mathrm{MnB}_{20}{ }^{+}, \mathrm{MoB}_{24}$, and $\mathrm{WB}_{24}$. However, the transition metal should possess both geometric and electronic states that can fit in high symmetric endohedral B cage-like clusters that eventually lead to the high stability structures that can be formed. The current endohedral $D_{2 \mathrm{~d}}$ $\mathrm{CrB}_{20}$ cage should be one such paradigm. The geometric factor responsible for the stability is straightforward. Our previous 
calculations have demonstrated that the $\mathrm{Cr}$ atom is too small to fit a large $\mathrm{B}_{24}$ cage, indicating smaller $\mathrm{B}$ cages are suitable for accommodating one $\mathrm{Cr}$ atom.

To understand electronic factors responsible for the stability of the endohedral $\mathrm{CrB}_{20}$ cage, the chemical bonding of the bare $D_{2 \mathrm{~d}} \mathrm{~B}_{20}$ cage and endohedral $D_{2 \mathrm{~d}} \mathrm{CrB}_{20}$ cage were analyzed based on canonical molecular orbitals (CMOs). Fig. 3 shows the comparison of eigenvalue spectra for the $D_{2 \mathrm{~d}}$ cage without (a) and with (b) Cr encapsulation. One can clearly note that the $\mathrm{Cr}$ encapsulation significantly increases the HOMO-LUMO gap from $0.87 \mathrm{eV}$ for the bare $D_{2 \mathrm{~d}} \mathrm{~B}_{20}$ cage to $4.38 \mathrm{eV}$ for the $D_{2 \mathrm{~d}}$ $\mathrm{CrB}_{20}$. For bare $D_{2 \mathrm{~d}} \mathrm{~B}_{20}$ (Fig. 3a), there are 6 occupied $\pi$-orbitals (HOMO, HOMO-1, HOMO-4, two HOMO-7, and HOMO-11) and 3 unoccupied $\pi$-orbitals (LUMO, LUMO+1 and LUMO+2). The moderate HOMO-LUMO gap in bare $D_{2 \mathrm{~d}} \mathrm{~B}_{20}$ is attributed to the mid-lying binding energies of these out-of-surface delocalized $\pi$-orbitals, and they will interact with the electronic orbitals of $\mathrm{Cr}$ atoms in the endohedral $\mathrm{CrB}_{20}$ cages. $\mathrm{Cr}$ has an electronic configuration of $[\mathrm{Ar}] 4 \mathrm{~s}^{1} 3 \mathrm{~d}^{5}$ with 6 valence electrons, adding the $12 \pi$-electrons (from 6 occupied $\pi$-orbitals) of bare $D_{2 \mathrm{~d}} \mathrm{~B}_{20}$ gives a total of 18 electrons. This special electron counting number of 18 is favorable for forming a stable 18electron closed-shell configuration, similar to that of previous $\mathrm{MnB}_{20}{ }^{+}, \mathrm{MoB}_{24}$ and $\mathrm{WB}_{24}$ clusters. Indeed, nine CMOs involved in the "spd- $\pi$ interaction" have been identified for the $D_{2 \mathrm{~d}}$ $\mathrm{CrB}_{20}$ endohedral cage as depicted in Fig. 3b, i.e., HOMO-13 (slike), HOMO-11 ( $\mathrm{d}_{x^{2}-y^{2}}$-like), HOMO-8 (double degenerate, $\mathrm{p}_{x^{-}}$ like and $\mathrm{p}_{y}$-like), HOMO-7 ( $\mathrm{d}_{x y}$-like), HOMO-6 ( $\mathrm{p}_{z}$-like), HOMO-5 (double degenerate, $\mathrm{d}_{x z}$-like and $\mathrm{d}_{y z}-\pi$ like) and HOMO-1 ( $3 \mathrm{~d}_{z^{2}}$-like). Thus, the $\mathrm{CrB}_{20}$ cluster represents another example of having a symmetric endohedral cage configuration stabilized by the 18 -electron configuration.

\section{Conclusions}

In summary, we systematically investigated the structural and electronic properties of $\mathrm{CrB}_{n}$ clusters with $n=8,10,12,14,16$, 18,20 and 22 through extensive swarm-intelligent structure searches and first-principles calculations. It is found that $\mathrm{Cr}$ doping significantly modifies the structural evolution of $\mathrm{B}$ clusters. Intriguing transitions from half-sandwich to drum-like and then to endohedral cage-like structures are revealed as the number of $\mathrm{B}$ atoms increases. $\mathrm{CrB}_{8}, \mathrm{CrB}_{10}$ and $\mathrm{CrB}_{12}$ clusters exhibit half-sandwich structure with quasi-planar B moieties similar to the bare B cluster, indicating that small-sized B clusters are promising inorganic ligands. A drum-like structure is formed with $\mathrm{CrB}_{16}$ clusters, while endohedral cage structures emerge with the larger $\mathrm{CrB}_{20}$ and $\mathrm{CrB}_{22}$ clusters. The endohedral $\mathrm{CrB}_{20}$ cage has a high symmetry of $D_{2 \mathrm{~d}}$ and the largest HOMO-LUMO gap among $\mathrm{CrB}_{n}$ in the current work, indicating its high chemical stability, which is attributed to the geometric fit between the size of the $\mathrm{Cr}$ atom and the void of the $\mathrm{B}$ cage as well as the formation of the 18-electron configuration.

\section{Conflicts of interest}

The authors declare no competing financial interest.

\section{Acknowledgements}

The authors acknowledge the funding support from National Natural Science Foundation of China (Grants No. 11534003, 11604117, 11404128, 11704151 and 61775081), the Science Challenge Project, No. TZ2016001, National Key Research and Development Program of China (Grant No. 2016YFB0201200), and Program for JLU Science and Technology Innovative Research Team. Part of the calculations were performed in the high performance computing center of Jilin University and Tianhe2-JK in the Beijing Computational Science Research Center.

\section{References}

1 X. Sun, X. Liu, J. Yin, J. Yu, Y. Li, Y. Hang, X. Zhou, M. Yu, J. Li, G. Tai and W. Guo, Adv. Funct. Mater., 2017, 27, 1603300.

2 B. Albert and H. Hillebrecht, Angew. Chem., Int. Ed., 2009, 48, 8640-8668.

3 F. Liu, C. Shen, Z. Su, X. Ding, S. Deng, J. Chen, N. Xu and H. Gao, J. Mater. Chem., 2010, 20, 2197.

4 V. Bezugly, J. Kunstmann, B. Grundkötter-Stock, T. Frauenheim, T. Niehaus and G. Cuniberti, ACS Nano, 2011, 5, 4997-5005.

5 J. Kunstmann, V. Bezugly, H. Rabbel, M. H. Rümmeli and G. Cuniberti, Adv. Funct. Mater., 2014, 24, 4127-4134.

6 G. Tai, T. Hu, Y. Zhou, X. Wang, J. Kong, T. Zeng, Y. You and Q. Wang, Angew. Chem., Int. Ed., 2015, 54, 15473-15477.

7 B. Feng, J. Zhang, Q. Zhong, W. Li, S. Li, H. Li, P. Cheng, S. Meng, L. Chen and K. Wu, Nat. Chem., 2016, 8, 563-568.

8 A. J. Mannix, X. F. Zhou, B. Kiraly, J. D. Wood, D. Alducin, B. D. Myers, X. Liu, B. L. Fisher, U. Santiago, J. R. Guest, M. J. Yacaman, A. Ponce, A. R. Oganov, M. C. Hersam and N. P. Guisinger, Science, 2015, 350, 1513-1516.

9 H. Tang and S. Ismail-Beigi, Phys. Rev. Lett., 2007, 99, 115501. 10 A. N. Alexandrova, A. I. Boldyrev, H. J. Zhai and L. S. Wang, Coord. Chem. Rev., 2006, 250, 2811-2866.

11 Z. Zhang, E. S. Penev and B. I. Yakobson, Chem. Soc. Rev., 2017, 46, 6746-6763.

12 A. P. Sergeeva, I. A. Popov, Z. A. Piazza, W. L. Li, C. Romanescu, L. S. Wang and A. I. Boldyrev, Acc. Chem. Res., 2014, 47, 1349-1358.

13 B. Kiran, S. Bulusu, H.-J. Zhai, S. Yoo, X. C. Zeng and L.-S. Wang, Proc. Natl. Acad. Sci., 2005, 102, 961-964.

14 E. Oger, N. R. M. Crawford, R. Kelting, P. Weis, M. M. Kappes and R. Ahlrichs, Angew. Chem., Int. Ed., 2007, 46, 8503-8506.

15 H. J. Zhai, Y. F. Zhao, W. L. Li, Q. Chen, H. Bai, H. S. Hu, Z. A. Piazza, W. J. Tian, H. G. Lu, Y. B. Wu, Y. W. Mu, G. F. Wei, Z. P. Liu, J. Li, S. D. Li and L. S. Wang, Nat. Chem., 2014, 6, 727-731.

16 J. Lv, Y. Wang, L. Zhu and Y. Ma, Nanoscale, 2014, 6, 1169211696.

17 T. B. Tai, L. Van Duong, H. T. Pham, D. T. T. Mai and M. T. Nguyen, Chem. Commun., 2014, 50, 1558-1560.

18 N. Gonzalez Szwacki, A. Sadrzadeh and B. I. Yakobson, Phys. Rev. Lett., 2007, 98, 166804. 
19 J. T. Muya, G. Gopakumar, M. T. Nguyen and A. Ceulemans, Phys. Chem. Chem. Phys., 2011, 13, 7524.

20 Q. Chen, W.-L. Li, Y.-F. Zhao, S.-Y. Zhang, H.-S. Hu, H. Bai, H.-R. Li, W.-J. Tian, H.-G. Lu, H.-J. Zhai, S.-D. Li, J. Li and L.-S. Wang, ACS Nano, 2015, 9, 754-760.

21 Q. Chen, S. Y. Zhang, H. Bai, W. J. Tian, T. Gao, H. R. Li, C. Q. Miao, Y. W. Mu, H. G. Lu, H. J. Zhai and S. D. Li, Angew. Chem., Int. Ed., 2015, 54, 8160-8164.

22 A. B. Rahane and V. Kumar, Nanoscale, 2015, 7, 4055-4062.

23 L. Sai, X. Wu, N. Gao, J. Zhao and R. B. King, Nanoscale, 2017, 9, 13905-13909.

24 F. Y. Tian and Y. X. Wang, J. Chem. Phys., 2008, 129, 024903.

25 T. B. Tai and M. T. Nguyen, Chem. Commun., 2016, 52, 16531656.

26 D. L. V. K. Prasad and E. D. Jemmis, Phys. Rev. Lett., 2008, 100, 100-103.

27 H. Li, N. Shao, B. Shang, L.-F. Yuan, J. Yang and X. C. Zeng, Chem. Commun., 2010, 46, 3878.

28 F. Li, P. Jin, D. E. Jiang, L. Wang, S. B. Zhang, J. Zhao and Z. Chen, J. Chem. Phys., 2012, 136, 074302.

29 C. Romanescu, T. R. Galeev, W. L. Li, A. I. Boldyrev and L. S. Wang, Acc. Chem. Res., 2013, 46, 350-358.

30 T. R. Galeev, C. Romanescu, W. L. Li, L. S. Wang and A. I. Boldyrev, Angew. Chem., Int. Ed., 2012, 51, 2101-2105.

31 I. A. Popov, W.-L. Li, Z. A. Piazza, A. I. Boldyrev and L.-S. Wang, J. Phys. Chem. A, 2014, 118, 8098-8105.

32 I. A. Popov, T. Jian, G. V. Lopez, A. I. Boldyrev and L. S. Wang, Nat. Commun., 2015, 6, 8654.

33 T. Jian, W. L. Li, I. A. Popov, G. V. Lopez, X. Chen, A. I. Boldyrev, J. Li and L. S. Wang, J. Chem. Phys., 2016, 144, 154310.

34 W.-L. Li, T. Jian, X. Chen, H.-R. Li, T.-T. Chen, X.-M. Luo, S.-D. Li, J. Li and L.-S. Wang, Chem. Commun., 2017, 53, 1587-1590.

35 L. Zhao, X. Qu, Y. Wang, J. Lv, L. Zhang, Z. Hu, G. Gu and Y. Ma, J. Phys. Condens. Matter, 2017, 29, 265401.

36 J. Lv, Y. Wang, L. Zhang, H. Lin, J. Zhao and Y. Ma, Nanoscale, 2015, 7, 10482-10489.

37 X. Dong, S. Jalife, A. Vásquez-Espinal, E. Ravell, S. Pan, J. L. Cabellos, W. Liang, Z. Cui and G. Merino, Angew. Chem., Int. Ed., 2018, 57, 4627-4631.

38 Y. Wang, J. Lv, L. Zhu and Y. Ma, Phys. Rev. B, 2010, 82, 094116.
39 J. Lv, Y. Wang, L. Zhu and Y. Ma, J. Chem. Phys., 2012, 137, 084104.

40 Y. Wang, J. Lv, L. Zhu and Y. Ma, Comput. Phys. Commun., 2012, 183, 2063-2070.

41 H. Wang, Y. Wang, J. Lv, Q. Li, L. Zhang and Y. Ma, Comput. Mater. Sci., 2016, 112, 406-415.

42 J. P. Perdew, K. Burke and M. Ernzerhof, Phys. Rev. Lett., 1996, 77, 3865-3868.

43 P. Li, X. Liu, M. Chen, P. Lin, X. Ren, L. Lin, C. Yang and L. He, Comput. Mater. Sci., 2016, 112, 503-517.

44 M. Chen, G. C. Guo and L. He, J. Phys. Condens. Matter, 2010, 22, 445501.

45 M. Chen, G. C. Guo and L. He, J. Phys. Condens. Matter, 2011, 23, 325501.

46 D. R. Hamann, Phys. Rev. B, 2013, 88, 085117.

47 M. J. Frisch, G. W. Trucks, H. B. Schlegel, G. E. Scuseria, M. A. Robb, J. R. Cheeseman, G. Scalmani, V. Barone, B. Mennucci, G. A. Petersson, H. Nakatsuji, M. Caricato, X. Li, H. P. Hratchian, A. F. Izmaylov, J. Bloino, G. Zheng, J. L. Sonnenberg, M. Hada, M. Ehara, K. Toyota, R. Fukuda, J. Hasegawa, M. Ishida, T. Nakajima, Y. Honda, O. Kitao, H. Nakai, T. Vreven, J. A. Montgomery, J. E. Peralta, F. Ogliaro, M. Bearpark, J. J. Heyd, E. Brothers, K. N. Kudin, V. N. Staroverov, R. Kobayashi, J. Normand, K. Raghavachari, A. Rendell, J. C. Burant, S. S. Iyengar, J. Tomasi, M. Cossi, N. Rega, J. M. Millam, M. Klene, J. E. Knox, J. B. Cross, V. Bakken, C. Adamo, J. Jaramillo, R. Gomperts, R. E. Stratmann, O. Yazyev, A. J. Austin, R. Cammi, C. Pomelli, J. W. Ochterski, R. L. Martin, K. Morokuma, V. G. Zakrzewski, G. A. Voth, P. Salvador, J. J. Dannenberg, S. Dapprich, A. D. Daniels, O. Farkas, J. B. Foresman, J. V. Ortiz, J. Cioslowski and D. J. Fox, Gaussian 09, Gaussian, Inc., Wallingford CT, 2009. 48 T. Lu and F. Chen, J. Comput. Chem., 2012, 33, 580-592.

49 C. Longjiu, J. Chem. Phys., 2012, 136, 104301.

50 T. Jian, W.-L. Li, X. Chen, T.-T. Chen, G. V. Lopez, J. Li and L.-S. Wang, Chem. Sci., 2016, 7, 7020-7027.

51 N. M. Tam, H. T. Pham, L. Van Duong, M. P. Pham-Ho and M. T. Nguyen, Phys. Chem. Chem. Phys., 2015, 17, 3000-3003. 52 D. Y. Zubarev and A. I. Boldyrev, Phys. Chem. Chem. Phys., 2008, 10, 5207.

53 E. D. Glendening, C. R. Landis and F. Weinhold, Wiley Interdiscip. Rev.: Comput. Mol. Sci., 2012, 2, 1-42. 